\title{
Mylohyoid Muscle
}

National Cancer Institute

\section{Source}

National Cancer Institute. Mylohyoid Muscle. NCI Thesaurus. Code C52962.

One of the suprahyoid muscles located superior to the anterior belly of the digastric muscle that has its origin on the mandible, its insertion on the hyoid bone, and functions to elevate the hyoid, the tongue, and the floor of the mouth. 